\title{
Time damping of linear non-adiabatic magnetohydrodynamic waves in an unbounded plasma with solar coronal properties
}

\author{
M. Carbonell ${ }^{1}$, R. Oliver $^{2}$, and J. L. Ballester ${ }^{2}$ \\ 1 Departament de Matemàtiques i Informàtica, Universitat de les Illes Balears, 07122 Palma de Mallorca, Spain \\ 2 Departament de Física, Universitat de les Illes Balears, 07122 Palma de Mallorca, Spain \\ e-mail: marc.carbonell@uib.es;ramon.oliver@uib.es
}

Received 29 July 2003 / Accepted 14 November 2003

\begin{abstract}
In this paper, we study the time damping of magnetoacoustic waves when the adiabaticity assumption is removed by means of an energy equation which includes optically thin radiative losses, thermal conduction and heating. For the sake of simplicity, this study has been done for a homogeneous, isothermal and unbounded medium permeated by a uniform magnetic field, with physical properties akin to those of the corona, the prominence-corona transition region (PCTR) and prominences. In some PCTR regimes and in the coronal regime wave instabilities appear, which prevents the computation of the damping time and the damping per period for part of the wavenumber interval considered. Furthermore, except for one of the PCTR regimes, in the rest of the regimes the apparition of those instabilities depends on the heating mechanism considered. Also, for the same heating mechanism, the behaviour of the damping time for the different considered regimes changes significantly when going from very small to very large wavenumbers and, in all the regimes, it becomes almost constant for very large wavenumbers.
\end{abstract}

Key words. Sun: oscillations - Sun: magnetic fields - Sun: corona - Sun: prominences

\section{Introduction}

During the last years, coronal seismology has undergone a strong growth due to the numerous observations of oscillations in coronal structures detected from ground and space-based instruments. For instance, oscillations have been detected in coronal loops (Nakariakov et al. 1999; Aschwanden et al. 1999; De Moortel et al. 2000; Robbrecht et al. 2001; Terradas 2002), in coronal holes (Ofman et al. 2000a; Banerjee et al. 2001), in coronal plumes (De Forest \& Gurman 1998; Banerjee et al. 2000) and in prominences (Engvold 2001; Terradas et al. 2002). Also, evidence for slow magnetosonic waves in coronal holes was found by Ofman et al. (1997), in coronal plumes by Ofman et al. (2000b) and in coronal loops, detected with the SUMER instrument, by Kliem et al (2002), Wang et al (2002) and Ofman \& Wang (2002). Some of these observations have been explained in terms of linear or non-linear magnetoacoustic waves including non-adiabatic terms. Linear nonadiabatic waves can provide with interesting physical effects such as time and spatial damping of disturbances, and the most simple approach is to take into consideration a radiative loss term in the energy equation based on Newton's law of cooling with a constant relaxation time $\left(\tau_{\mathrm{r}}\right)$. Then, the modes may have complex frequencies and so perturbations contain a term of the form $\exp \left(-t / \tau_{\mathrm{d}}\right)$, where $\tau_{\mathrm{d}}$ is the damping time. The limit $\tau_{\mathrm{r}} \rightarrow \infty$ corresponds to a perturbation that takes infinite time

Send offprint requests to: J. L. Ballester,

e-mail: dfsjlbo@uib.es to damp, i.e. the adiabatic limit, while $\tau_{\mathrm{r}}=0$ corresponds to perturbations which are immediately damped, i.e. the isothermal limit. For $0<\tau_{\mathrm{r}}<\infty$ the situation is intermediate between the isothermal and the adiabatic limits and heat is exchanged between different locations at finite speed (Mihalas $\&$ Mihalas 1984). In the case of a magnetised medium, this approach has been used by Webb \& Roberts (1980), who analysed magnetohydrodynamic waves in an unbounded atmosphere in the presence of a uniform vertical magnetic field, by Bünte \& Bogdan (1994), who studied magneto-atmospheric waves in infinite and semi-infinite stratified, isothermal atmospheres, and by Banerjee et al. (1997) who have considered the effects of radiation losses on magneto-atmospheric-gravity waves in a stratified, magnetised atmosphere having a vertical magnetic field and bounded in the vertical direction.

A more complete approach to linear non-adiabatic waves can be obtained with the inclusion of different mechanisms representing radiative losses and heating, as well as including thermal conduction. In this regard, Ibañez \& Escalona (1993) studied the spatial damping and amplification of magnetosonic linear waves in an optically thin plasma with solar abundances in the temperature range $10^{4}-10^{8} \mathrm{~K}$. Furthermore, using nonadiabatic MHD waves, several studies have focused on the problem of coronal heating by proposing that such heating can be provided by means of different mechanisms based on wave dissipation. For instance, Porter et al. (1994a) investigated the role of high-frequency waves in the heating of solar active region coronal loops, by considering the ion compressive 
viscosity as the damping mechanism for waves, and Porter et al. (1994b) studied the damping rate and length of non-adiabatic waves, subject to dissipation by viscosity and thermal conduction in an unbounded magnetic plasma, as a mechanism to heat the solar corona.

However, we must keep in mind that in many cases the study of non-linear MHD waves in the solar corona is of interest since non-linearity combined with different types of dissipation can explain some observational features detected in the propagation of magnetoacoustic or Alfvén waves in coronal structures (Nakariakov et al. 2000a). For instance, Ofman et al. (1999) have used nonlinear analytical and numerical approaches to study the damping of slow magnetosonic waves in coronal plumes while the damping of non-adiabatic magnetosonic waves in loops has been studied numerically by Ofman \& Wang (2002).

It is well known that solar prominences are embedded in the solar corona and have a prominence-corona transition region (PCTR) in which the density and temperature vary from prominence to coronal values and, although non-linear effects could be important in those regions, observations of oscillations in prominences and in the surrounding plasma suggest that they are of small amplitude and thus can be interpreted in terms of linear MHD waves. Furthermore, time damping of oscillations in prominences has been reported by Molowny-Horas et al. (1999) and Terradas et al. (2002), while radiative damping of these oscillations, using the Newton's law of cooling with constant relaxation time, has been explored by Terradas et al. (2001). However, a more in-depth study would require to include an energy equation in which radiation, heating and thermal conduction should be considered. For this reason, in this work our aim is to perform a first approach to the time damping of linear non-adiabatic magnetoacoustic waves in unbounded media having properties akin to those of the solar corona, the prominence-corona transition region and quiescent prominences. As the background model, we use a homogeneous unbounded medium threaded by a constant and horizontal magnetic field, and we have considered an optically thin energy equation including radiative losses, thermal conduction, as well as different expressions, representing various mechanisms, for the heating term.

The layout of the paper is as follows: In Sect. 2, the equilibrium model, the linearised equations and the general dispersion relation are presented; in Sect. 3 the general dispersion relation is solved and the results for the different regimes and heating mechanisms are shown; finally, in Sect. 4, conclusions are drawn.

\section{Basic equations}

As the equilibrium configuration we consider a homogeneous slab that is infinite in all directions and is threaded by a uniform magnetic field along the $x$-direction, say. The equilibrium magnitudes of the slab are given by

$p_{0}=$ const., $\rho_{0}=$ const., $T_{0}=$ const., $\boldsymbol{B}_{0}=B_{0} \hat{\boldsymbol{e}}_{x}, \boldsymbol{v}_{0}=\mathbf{0}$,

with $B_{0}=$ constant, and the effect of gravity has been ignored.
The basic MHD equations for the discussion of linear waves are:

$$
\begin{aligned}
& \frac{\mathrm{D} \rho}{\mathrm{D} t}+\rho \nabla \cdot v=0 \\
& \rho \frac{\mathrm{D} \boldsymbol{v}}{\mathrm{D} t}=-\nabla p+\frac{1}{\mu}(\nabla \times \boldsymbol{B}) \times \boldsymbol{B}+\rho g, \\
& \rho T \frac{\mathrm{D} s}{\mathrm{D} t}+\rho L(\rho, T)-\nabla \cdot(\kappa \cdot \nabla T)=0, \\
& \frac{\partial \boldsymbol{B}}{\partial t}=\nabla \times(\boldsymbol{v} \times \boldsymbol{B}), \\
& \nabla \cdot \boldsymbol{B}=0 \\
& p=\frac{\rho R T}{\tilde{\mu}}
\end{aligned}
$$

where $\frac{\mathrm{D}}{\mathrm{D} t}=\frac{\partial}{\partial t}+\boldsymbol{v} \cdot \nabla$ is the material derivative for time variations following the motion. In Eq. (4), the term $\nabla \cdot(\kappa \cdot \nabla T)$ represents the thermal conduction, although in our case perpendicular thermal conduction has been neglected, and $L$ is the heat-loss function which depends on the local plasma parameters. In the case of an equilibrium with uniform temperature, such as we consider here, the heat-loss function is

$$
L\left(\rho_{0}, T_{0}\right)=0 .
$$

Usually, in solar applications this function represents the difference between an arbitrary heat input and a radiative loss function which, in our case, has been chosen as the optically thin radiative loss function (Hildner 1974). Then, our heat-loss function is given by

$L(\rho, T)=\chi^{*} \rho T^{\alpha}-h \rho^{a} T^{b}$,

$\chi^{*}$ and $\alpha$ being piecewise functions depending on the temperature (Hildner 1974). The use of an optically thin plasma radiative cooling seems to be a reasonable approach for coronal, or almost coronal, conditions, while it may not be valid for prominence conditions because they are optically thick. In this case, the radiative losses from the internal part of the prominence are greatly reduced and this can be represented by changing the exponent $\alpha$ in the cooling function, for temperatures $T \leq 10^{4} \mathrm{~K}$, from $\alpha=7.4$ to 17.4 (Milne et al. 1979) or $\alpha=30$ (Rosner et al. 1978), as well as by changing $\chi^{*}$ accordingly. Finally, the last term in Eq. (8) represents an arbitrary heating function which can be modified by taking different values for the exponents $a$ and $b$. In our case, different heating scenarios have been considered, and the values taken into account for exponents $a$ and $b$ in Eq. (8) are (Rosner et al. 1978; Dahlburg \& Mariska 1988)

1. constant heating per unit volume $(a=b=0)$;

2. constant heating per unit mass $(a=1, b=0)$;

3 . heating by coronal current disipation $(a=1, b=1)$;

4. heating by Alfvén mode/mode conversion $(a=b=7 / 6)$;

5. heating by Alfvén mode/anomalous conduction damping $(a=1 / 2, b=-1 / 2)$. 
Considering small perturbations from the equilibrium in the form

$\boldsymbol{B}(t, \boldsymbol{r})=\boldsymbol{B}_{0}+\boldsymbol{B}_{1}(t, \boldsymbol{r}), \quad p(t, \boldsymbol{r})=p_{0}+p_{1}(t, \boldsymbol{r})$,

$\rho(t, \boldsymbol{r})=\rho_{0}+\rho_{1}(t, \boldsymbol{r}), T(t, \boldsymbol{r})=T_{0}+T_{1}(t, \boldsymbol{r}), \boldsymbol{v}(t, \boldsymbol{r})=\boldsymbol{v}_{1}(t, \boldsymbol{r})$

we linearise the basic Eqs. (2)-(7) to obtain

$\frac{\partial \rho_{1}}{\partial t}+\rho_{0} \nabla \cdot \boldsymbol{v}=0$

$\rho_{0} \frac{\partial v}{\partial t}=-\nabla p_{1}+\frac{1}{\mu}\left(\boldsymbol{B}_{0} \cdot \nabla\right) \boldsymbol{B}_{1}-\frac{1}{\mu} \nabla\left(\boldsymbol{B}_{0} \cdot \boldsymbol{B}_{1}\right)$,

$\frac{\partial p_{1}}{\partial t}-c_{\mathrm{s}}^{2} \frac{\partial \rho_{1}}{\partial t}=(\gamma-1)\left(\boldsymbol{B}_{0} \cdot \nabla\right)\left[\frac{\kappa_{\|}}{B_{0}^{2}}\left(\boldsymbol{B}_{0} \cdot \nabla\right) T_{1}\right]$ $-(\gamma-1)\left(L+\rho_{0} L_{\rho}\right) \rho_{1}-(\gamma-1) \rho_{0} L_{T} T_{1}$,

$\frac{\partial \boldsymbol{B}_{1}}{\partial t}=\nabla \times\left(\boldsymbol{v} \times \boldsymbol{B}_{0}\right)$,

$\nabla \cdot \boldsymbol{B}_{1}=0$

$\frac{p_{1}}{p_{0}}-\frac{\rho_{1}}{\rho_{0}}-\frac{T_{1}}{T_{0}}=0$

where $\kappa_{\|}=10^{-11} T^{5 / 2}$. Now, since the medium is unbounded we can perform a Fourier analysis in plane waves and assume perturbations behaving like $\mathrm{e}^{\mathrm{i}(\omega t-\boldsymbol{k} \cdot \boldsymbol{r})}$, and with no loss of generality we choose the $z$-axis so that the wavevector $\boldsymbol{k}$ lies in the $x z$-plane, so that

$\boldsymbol{k}=k_{x} \hat{\boldsymbol{e}}_{x}+k_{z} \hat{\boldsymbol{e}}_{z}$.

Then, the following scalar equations are obtained

$\omega \rho_{1}-\rho_{0}\left(k_{x} v_{x}+k_{z} v_{z}\right)=0$,

$\omega \rho_{0} v_{x}-k_{x} p_{1}=0$,

$\omega \rho_{0} v_{y}+\frac{1}{\mu} B_{0 x} k_{x} B_{1 y}=0$,

$\omega \rho_{0} v_{z}-k_{z} p_{1}+\frac{1}{\mu} B_{0 x}\left(k_{x} B_{1 z}-k_{z} B_{1 x}\right)=0$,

$\omega B_{1 x}-B_{0 x} k_{z} v_{z}=0$,

$\omega B_{1 y}+B_{0 x} k_{x} v_{y}=0$

$\omega B_{1 z}+B_{0 x} k_{x} v_{z}=0$,

$\frac{p_{1}}{p_{0}}-\frac{\rho_{1}}{\rho_{0}}-\frac{T_{1}}{T_{0}}=0$,

$$
\begin{gathered}
\mathrm{i} \omega p_{1}-\mathrm{i} \omega c_{\mathrm{s}}^{2} \rho_{1}+(\gamma-1)\left(\kappa_{\|} k_{x}^{2}+\rho_{0} L_{T}\right) T_{1} \\
+(\gamma-1)\left(L+\rho_{0} L_{\rho}\right) \rho_{1}=0 .
\end{gathered}
$$

Equations (17) and (20) are decoupled from the rest and describe Alfvén waves, which are not investigated here. Next, we have eliminated the perturbations $p_{1}, T_{1}, \rho_{1}, B_{1 x}, B_{1 z}$ in favour of $v_{x}, v_{z}$, thus obtaining two algebraic equations for the velocity perturbations. Then, imposing that the determinant of this algebraic system be zero and factoring out the expression $-B_{0}^{2} k_{x}^{2}+\mu \omega^{2} \rho_{0}$, we obtain our general dispersion relation,

$a_{5} \omega^{5}+a_{4} \omega^{4}+a_{3} \omega^{3}+a_{2} \omega^{2}+a_{1} \omega+a_{0}=0$,

whose coefficients are given by:

$a_{0}=\left[\mathrm{i} B_{0}^{2} k_{x}^{2} k^{2} p_{0}\left(-A T_{0}+H \rho_{0}\right)\right] / \mu \rho_{0}^{3}$,

$a_{1}=B_{0}^{2} c_{\mathrm{s}}^{2} k_{x}^{2} k^{2} p_{0} / \mu \rho_{0}^{2}$,

$a_{2}=\left[\mathrm{i} k^{2}\left(A B_{0}^{2} T_{0}+\mu p_{0}\left(A T_{0}-H \rho_{0}\right)\right)\right] / \mu \rho_{0}^{2}$,

$a_{3}=-\left[k^{2} p_{0}\left(B_{0}^{2}+\mu c_{\mathrm{s}}^{2} \rho_{0}\right)\right] / \mu \rho_{0}^{2}$,

$a_{4}=-\mathrm{i} A T_{0} / \rho_{0}$,

$a_{5}=p_{0} / \rho_{0}$,

with

$A=(\gamma-1) \kappa_{\|} k_{x}^{2}+(\gamma-1) \rho_{0} L_{T}$,

$H=(\gamma-1)\left(L+\rho_{0} L_{\rho}\right)$.

In both expressions, the factors $L_{\rho}, L_{T}$ are:

$L_{\rho}=\left(\frac{\partial L}{\partial \rho}\right)_{T}$

and

$L_{T}=\left(\frac{\partial L}{\partial T}\right)_{p}$,

with $T$ and $p$ held constant, respectively, at the equilibrium state.

Taking $k_{x}=k \cos \theta$ and $k_{z}=k \sin \theta$, the resulting dispersion relation can be written as

$$
\begin{gathered}
\omega^{5}-\frac{\mathrm{i} A T_{0}}{p_{0}} \omega^{4}-k^{2}\left(c_{\mathrm{a}}^{2}+c_{\mathrm{s}}^{2}\right) \omega^{3}+\mathrm{i} k^{2}\left(\frac{A T_{0} c_{\mathrm{a}}^{2}}{p_{0}}+\frac{A T_{0}-H \rho_{0}}{\rho_{0}}\right) \omega^{2} \\
+c_{\mathrm{a}}^{2} c_{\mathrm{s}}^{2} k^{4} \cos ^{2} \theta \omega+\frac{\mathrm{i} c_{\mathrm{a}}^{2} k^{4}\left(H \rho_{0}-A T_{0}\right) \cos ^{2} \theta}{\rho_{0}}=0 .
\end{gathered}
$$

Equation (26) can also be written in terms of the quantities $k_{T}^{\prime}$ and $k_{\rho}$ introduced by Field (1965) in his study of thermal instabilities and which are defined as

$k_{\rho}=\frac{\mu(\gamma-1) \rho_{0} L_{\rho}}{R c_{\mathrm{s}} T_{0}}$

$k_{T}^{\prime}=k_{T}+\frac{\kappa_{\|}^{2}}{k_{k_{\|}}}+\frac{\kappa_{\perp}^{2}}{k_{\kappa_{\perp}}}$

with

$k_{T}=\frac{\mu(\gamma-1) L_{T}}{R c_{\mathrm{s}}}$

$k_{K}=\frac{R c_{\mathrm{s}} \rho_{0}}{\mu(\gamma-1) \kappa}$.

Here $k_{\kappa}^{-1}$ is the mean free path of the conducting particles, while $k_{\rho}$ and $k_{T}$ are the wavenumbers of sound waves whose angular frequencies are numerically equal to the growth rates 
Table 1. Parameter values considered in the different studied regimes representing prominence, PCTR and solar corona physical conditions. All quantities are expressed in MKS units.

\begin{tabular}{ccccccc}
\hline \hline Regime & $T$ & $\rho$ & $\chi^{*}$ & $\alpha$ & $\tilde{\mu}$ & Reference \\
\hline Prominence (1.1) & 8000 & $5 \times 10^{-11}$ & $1.76 \times 10^{-13}$ & 7.4 & 0.8 & Hildner (1974) \\
Prominence (1.2) & 8000 & $5 \times 10^{-11}$ & $1.76 \times 10^{-53}$ & 17.4 & 0.8 & Milne et al. (1979) \\
Prominence (1.3) & 8000 & $5 \times 10^{-11}$ & $7.01 \times 10^{-104}$ & 30 & 0.8 & Rosner et al. (1978) \\
PCTR (2) & 50000 & $10^{-12}$ & $4.29 \times 10^{10}$ & 1.8 & 0.5 & Hildner (1974) \\
PCTR (3) & 200000 & $10^{-13}$ & $2.86 \times 10^{19}$ & 0 & 0.5 & Hildner (1974) \\
PCTR (4) & 500000 & $5 \times 10^{-13}$ & $1.41 \times 10^{33}$ & -2.5 & 0.5 & Hildner (1974) \\
Corona (5) & 1000000 & $5 \times 10^{-14}$ & $1.97 \times 10^{24}$ & -1 & 0.5 & Hildner (1974) \\
\hline
\end{tabular}

of isothermal and isochoric perturbations, respectively; in the presence of a magnetic field, $k_{T}^{\prime}$ corresponds to $k_{T}$ modified by conduction effects. Using these quantities and the above expressions of $A$ and $H$, we obtain

$k_{T}^{\prime}=\frac{A T_{0}}{p_{0} c_{\mathrm{s}}}$

and

$k_{\rho}=\frac{H \rho_{0}}{p_{0} c_{\mathrm{s}}}$.

Now, Eq. (26) becomes

$$
\begin{gathered}
\omega^{5}-\mathrm{i} c_{\mathrm{s}} k_{T}^{\prime} \omega^{4}-k^{2}\left(c_{\mathrm{a}}^{2}+c_{\mathrm{s}}^{2}\right) \omega^{3}+\mathrm{i} k^{2}\left[\frac{c_{\mathrm{s}}^{3}\left(k_{T}^{\prime}-k_{\rho}\right)}{\gamma}+c_{\mathrm{a}}^{2} c_{\mathrm{s}} k_{T}^{\prime}\right] \omega^{2} \\
+c_{\mathrm{a}}^{2} c_{\mathrm{s}}^{2} k^{4} \cos ^{2} \theta \omega+\mathrm{i}\left(\frac{k_{\rho}-k_{T}^{\prime}}{\gamma}\right) c_{\mathrm{a}}^{2} c_{\mathrm{s}}^{3} k^{4} \cos ^{2} \theta=0
\end{gathered}
$$

A simple check of this general dispersion relation can be performed by setting $k_{T}^{\prime}=k_{\rho}=0$, equivalent to $(A=H=0)$, in Eq. (27), so we obtain:

$\omega^{4}-\left(c_{\mathrm{a}}^{2}+c_{\mathrm{s}}^{2}\right) k^{2} \omega^{2}+c_{\mathrm{a}}^{2} c_{\mathrm{s}}^{2} k^{4} \cos ^{2} \theta=0$,

which is the well-known dispersion relation for adiabatic magnetoacoustic waves.

With proper choices for the density, temperature and magnetic field, the different considered regimes can be used to mimic the physical properties of prominences, the prominencecorona transition region (PCTR) and the solar corona. The variation of the temperature between the different regimes implies that the values of the piecewise constants $\chi^{*}$ and $\alpha$, in the optically thin cooling function, must be modified (Table 1). The first regime corresponds to quiescent prominence conditions, the following three regimes correspond to physical conditions typical of the PCTR, while the last regime corresponds to solar coronal conditions. For each of the considered regimes the five different heating mechanisms, listed above, have been taken into account. Then, for a magnetic field of $10 \mathrm{G}$ and a propagation angle of $\pi / 4$, the dispersion relation Eq. (27) has been solved numerically and since we are interested in the time damping of magnetoacoustic waves we consider the wavenumber, $k$, to be real and seek for complex solutions of the frequency $\omega$ expressed as $\omega=\omega_{\mathrm{R}}+\mathrm{i} \omega_{\mathrm{I}}$. Equation (27) is a fifth order polynomial for $\omega$ as a function of $k$ and, such as can be seen in Appendix A, we obtain one purely imaginary root, corresponding to the thermal or condensation mode, while the rest of the roots, corresponding to wave modes, consist of two pairs: one pair represents the slow mode, whereas the other represents the fast mode. For each pair $\left(\omega_{1}, \omega_{2}\right), \omega_{1}=\omega_{R}+i \omega_{I}$ and $\omega_{2}=-\omega_{\mathrm{R}}+\mathrm{i} \omega_{\mathrm{I}}$. Then, the period of the waves is given by $T=\frac{2 \pi}{\omega_{\mathrm{R}}}$, the damping time is $\tau_{\mathrm{D}}=\frac{1}{\omega_{\mathrm{I}}}$ and the damping per period is $D_{\mathrm{p}}=\frac{\omega_{\mathrm{I}}}{\omega_{\mathrm{R}}}$. On the other hand, the thermal or condensation mode has not been considered in our study since this mode does not represent a propagating wave and, in addition, thermal instabilities were extensively studied by Field (1965).

\section{Results}

\subsection{Regime 1 (Prominence conditions)}

First of all, we have compared the results obtained for the three prominence regimes considered when the different heating mechanisms are taken into account. In the prominence regime no thermal instability appears and within each prominence regime the behaviour of fast and slow waves is similar when different heating mechanisms are considered. Therefore, from now on, and for the prominence regime, we have taken into account only one of the heating mechanisms. Next, we have studied the differences in the behaviour of the slow and fast waves produced by the change in the parameters $\chi^{*}$ and $\alpha$ of the different prominence regimes. The idea behind the modification of these parameters is that prominences are optically thick, so radiative losses from the inner parts are greatly reduced and the values for $\chi^{*}$ and $\alpha$ considered in Table 1 reduce the losses by two orders of magnitude when going from regime 1.1 to 1.3 . Figure 1 shows that while the period of waves is not affected by the variation of these parameters, the damping time and the damping per period are modified only for wavenumbers between $10^{-10}$ and $10^{-3} \mathrm{~m}^{-1}$. For instance, in the region of wavenumbers between $10^{-10}$ and $10^{-7} \mathrm{~m}^{-1}$, and for a fixed $k$, the damping time decreases by two orders of magnitude when radiative losses are reduced, while the opposite happens in the region of wavenumbers between $10^{-7}$ and $10^{-3} \mathrm{~m}^{-1}$.

In Fig. 2, we show the results obtained for regime 1.1 with the values of density and temperature shown in Table 1 . This figure points out that the period of both waves decreases linearly when going from very small to very large wavenumbers, 

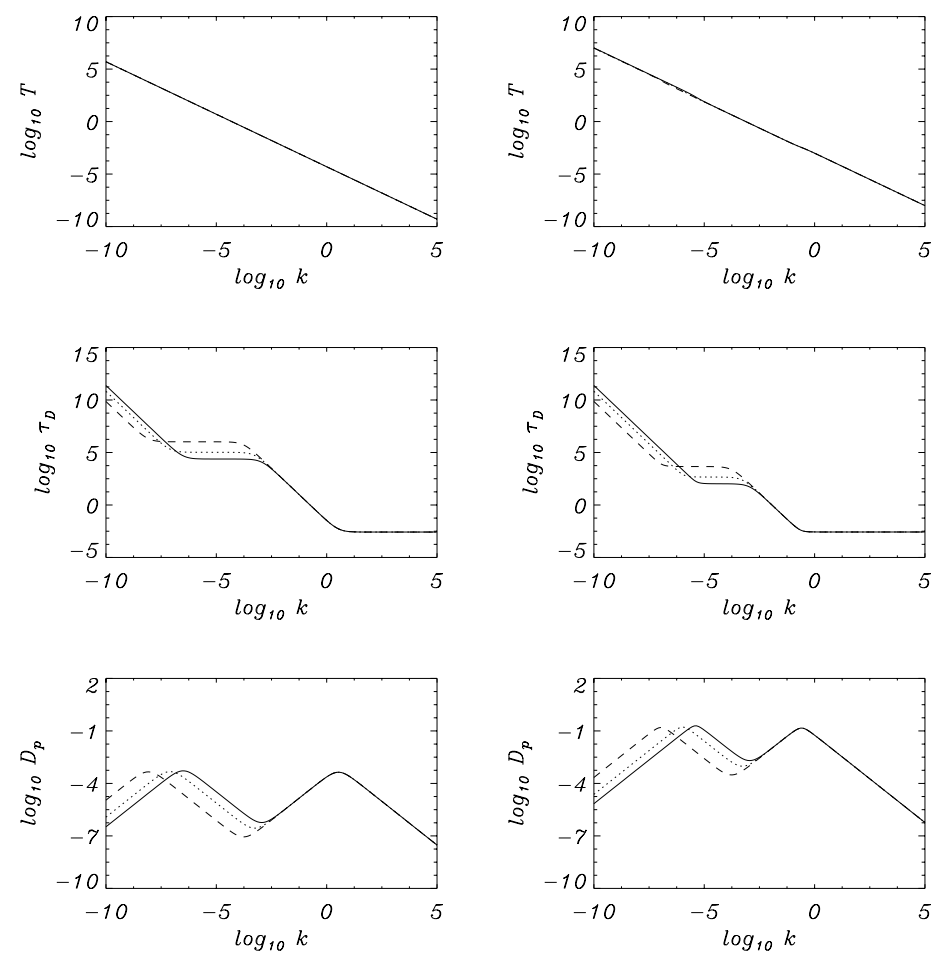

Fig. 1. Period $(T)$, damping time $\left(\tau_{\mathrm{D}}\right)$ and damping per period $\left(D_{\mathrm{p}}\right)$ for the fast $($ left $)$ and slow (right) waves in the three prominence regimes with a heating mechanism characterised by $a=b=0$. Continuous, dotted and dashed lines correspond to prominence regimes characterised by: $\alpha=7.4, \chi^{*}=1.76 \times 10^{-13} ; \alpha=17.4, \chi^{*}=1.76 \times 10^{-53} ; \alpha=30, \chi^{*}=7.01 \times 10^{-104}$, respectively.

and by performing a linear regression it is found that, both for fast and slow waves, the period behaves as $T \propto k^{-1}$, so both waves are very dispersive. On the other hand, the damping time also decreases when going from very small to large wavenumbers but two peculiar features, in the form of almost flat regions, appear for the wavenumber intervals $k=10^{-7}-10^{-4} \mathrm{~m}^{-1}$ and $k=10^{-1}-10^{5} \mathrm{~m}^{-1}$.

In the case of wavenumbers between $10^{-10}$ and $10^{-7} \mathrm{~m}^{-1}$, the behaviour of the damping time can be easily understood since in this region the coefficients $k_{\rho}$ and $k_{T}^{\prime}$ are very small, which means that we are almost in the adiabatic regime and, so, the imaginary parts of the frequencies of the magnetoacoustic modes are very small. On the other hand, by performing the approximation of very small $k$ in Eq. (27), i.e. neglecting the terms with $k$, we are left with an approximate expression for the frequency of the thermal mode,

$\omega=\mathrm{i} c_{\mathrm{s}} k_{T}^{\prime}$,

which exactly reproduces, in this region, the solution given by the full numerical solution of Eq. (27). Then, in this region we are still close to the adiabatic regime, so that the imaginary frequency of the thermal mode is very small and the real parts of the frequencies of the magnetoacoustic modes are similar to those of the adiabatic case, while their imaginary parts are very small.

When we enter the region of $k$ between $10^{-7}$ and $10^{-3} \mathrm{~m}^{-1}$ the behaviour of the damping time changes drastically. This behaviour can be understood as follows: For each heating mechanism considered, the coefficient $k_{\rho}$ is small (or zero) but constant, while the first term of $k_{T}^{\prime}$ (see Eq. (24)), which includes the horizontal wavenumber $k_{x}$ and the thermal conduction effects, starts to grow, which means that the thermal conduction starts to play a significant role and we enter a fully nonadiabatic regime. In this situation, $k_{T}^{\prime}$ and the imaginary parts of the frequencies of the magnetoacoustic modes grow slowly, and an almost flat region appears in the plot of the damping time versus wavenumber. The damping time of slow and fast waves is almost constant although there is a difference of two orders of magnitude between both.

For the interval of $k$ between $10^{-3}$ and $10^{0} \mathrm{~m}^{-1}$, there is a rapid decrease in the damping time, since the imaginary parts of the frequencies of the magnetoacoustic waves increase, which is probably due to a rapid increase of $k_{T}^{\prime}$ with $k_{x}$, so the effect of thermal conduction becomes more important.

Finally, a second flat region appears since, starting from $k$ around $10^{0} \mathrm{~m}^{-1}$, the imaginary parts of the frequencies of the magnetoacoustic waves become constant.

The behaviour of the damping time versus the wavenumber $k$ can be also understood in terms of the evolution, with $k$, of the coefficients of Eq. (27). In the first region, the coefficients of $\omega^{5}$ and $\omega^{4}$ are dominant and the rest can be neglected, so the frequency of the thermal mode is obtained using this approximate dispersion relation while the frequencies of the magnetoacoustic waves are almost those of the adiabatic case. In the second region, between $k=10^{-7}$ and $10^{-3} \mathrm{~m}^{-1}$, the situation reverses: first all the coefficients attain similar values and later the coefficients of $\omega^{2}$ and the independent term become dominant. In the third region, this dominance is still clearer and in the fourth region, the coefficient of $\omega^{5}$ is negligible as compared to the rest and making this approximation and solving 

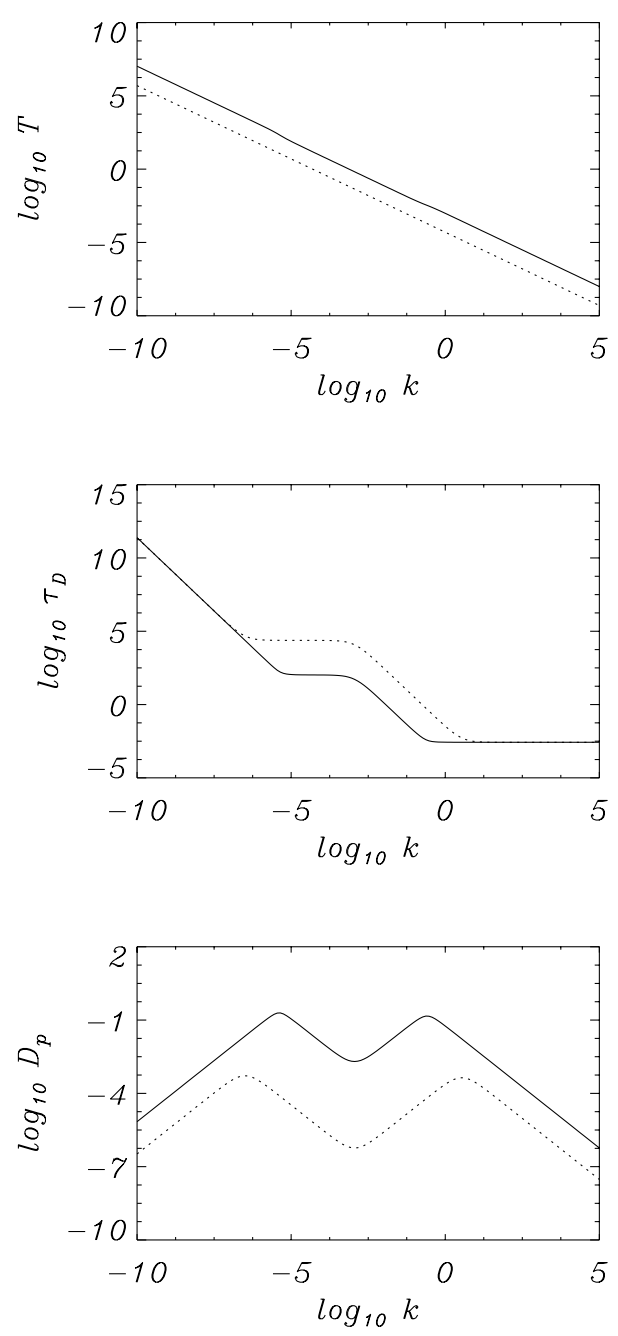

Fig. 2. Period $(T)$, damping time $\left(\tau_{\mathrm{D}}\right)$ and damping per period $\left(D_{\mathrm{p}}\right)$ for the fast (dotted) and slow (continuous) waves in the prominence regime 1.1 , with the heating mechanism characterised by $a=b=0$.

a fourth order dispersion relation in $\omega$ we obtain the constant damping time shown in the second flat region in Fig. 2.

Furthermore, in order to ascertain the influence of different parameters on the damping time, we have modified the values of the magnetic field, the density and the temperature, always within a range typical of prominences, as well as the value of the angle of propagation.

Figure 3 shows the behaviour of the slow wave when the temperature of the prominence regime is modified. The most important variation affects the damping time and it can be appreciated that for wavenumbers between $10^{-7}$ and $10^{-3} \mathrm{~m}^{-1}$ the damping time of the slow waves increases by two orders of magnitude when the temperature varies from $5000 \mathrm{~K}$ to $10000 \mathrm{~K}$; the same happens with fast waves.

Figure 4 shows the behaviour of fast waves when the density is modified. For wavenumbers between $10^{-7}$ and $10^{-3} \mathrm{~m}^{-1}$ or greater than $10^{0} \mathrm{~m}^{-1}$ an increase in the density produces a decrease of the damping time of the fast wave. A similat but no so important behaviour is found for the slow wave.

Furthermore, we have varied the angle of propagation, finding that the most important modification of the behaviour
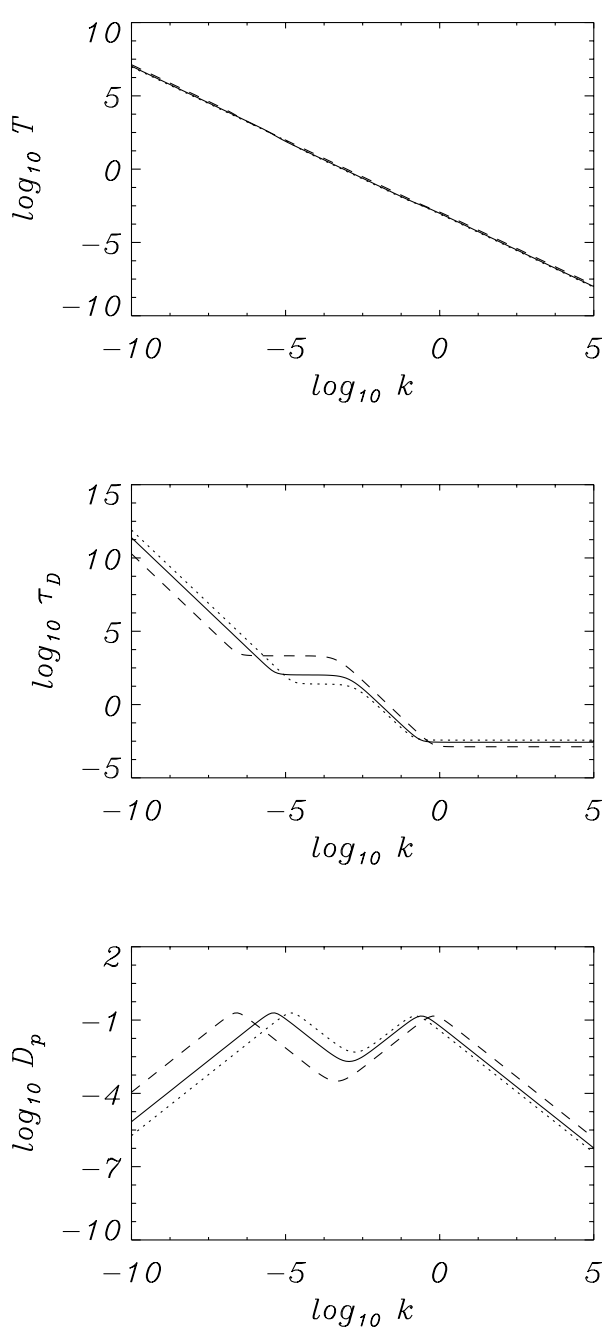

Fig. 3. Period $(T)$, damping time $\left(\tau_{\mathrm{D}}\right)$ and damping per period $\left(D_{\mathrm{p}}\right)$ of the slow wave in the prominence regime 1.1 , with the heating mechanism characterised by $a=b=0$, and three different temperatures, $T_{0}=5000 \mathrm{~K}$ (dashed), $8000 \mathrm{~K}$ (continuous) and $10000 \mathrm{~K}$ (dotted).

appears for $\pi / 2$. In this case, Eq. (27) becomes:

$$
\begin{aligned}
& \omega^{3}-\mathrm{i} c_{\mathrm{s}} k_{T}^{\prime} \omega^{2}-k^{2}\left(c_{\mathrm{a}}^{2}+c_{\mathrm{s}}^{2}\right) \omega \\
& +\mathrm{i} k^{2}\left[\frac{c_{\mathrm{s}}^{3}\left(k_{T}^{\prime}-k_{\rho}\right)}{\gamma}+c_{\mathrm{a}}^{2} c_{\mathrm{s}} k_{T}^{\prime}\right]=0
\end{aligned}
$$

and the slow wave does not propagate. Regarding the fast wave, we find that for very small wavenumbers the behaviour is as in the previous cases but when a wavenumber around $10^{-6} \mathrm{~m}^{-1}$ is attained the time damping becomes almost constant. In this case the first term of $A$ (Eq. (24)) is zero since thermal conduction does not play any role, this results in $k_{T}^{\prime}$ independent of the wavenumber and only the coefficients of the third and fourth terms of Eq. (28) vary with $k$. For large wavenumbers, the first and second terms of the equation can be neglected and the frequency of the thermal mode is given by the ratio between the coefficients of the remaining two terms and is independent of the wavenumber. Knowing this frequency we can factorise the dispersion relation and obtain a second order polynomial which once solved indicates that the real part of the fast mode 

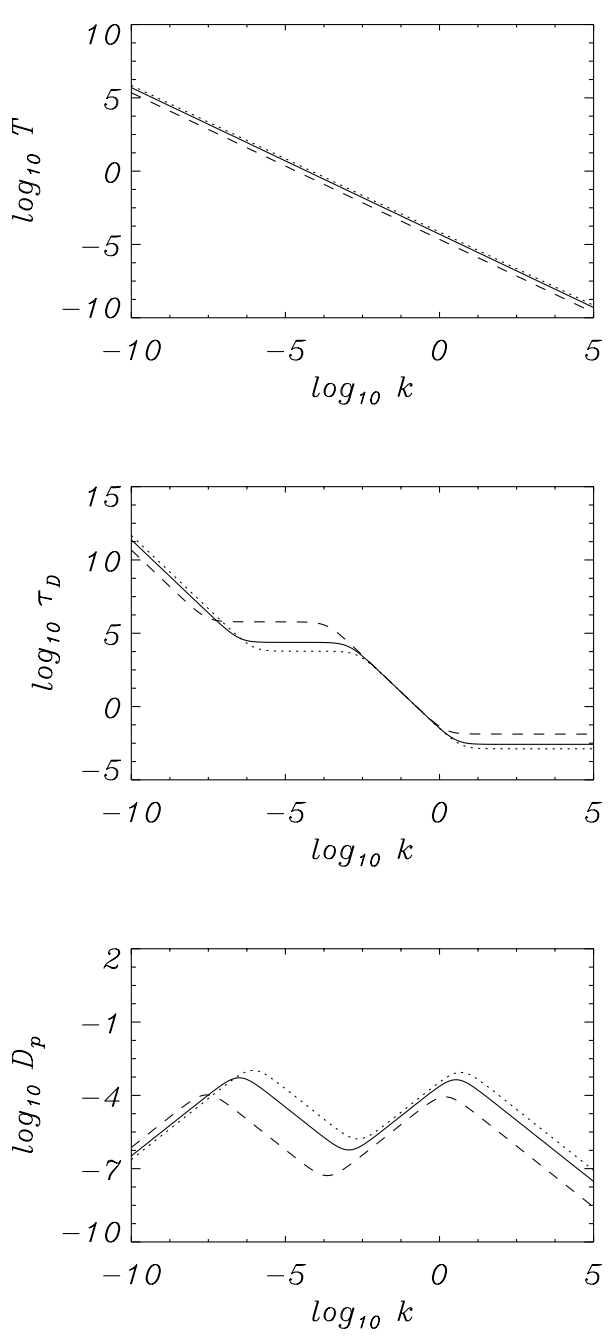

Fig. 4. Period $(T)$, damping time $\left(\tau_{\mathrm{D}}\right)$ and damping per period $\left(D_{\mathrm{p}}\right)$ of the fast wave in the prominence regime 1.1, with the heating mechanism characterised by: $a=b=0$, and three different densities, $\rho=10^{-11} \mathrm{~g} \mathrm{~cm}^{-3}$ (dashed), $\rho=5 \times 10^{-11} \mathrm{~g} \mathrm{~cm}^{-3}$ (continuous) and $\rho=10^{-10} \mathrm{~g} \mathrm{~cm}^{-3}$ (dotted).

frequency depends on the wavenumber, while the imaginary part is wavenumber independent, such as we obtain in the numerical results.

Finally, the variation of the magnetic field strength does not affect the behaviour of the slow wave and has a very small effects on the fast wave.

\subsection{Regime 2 (PCTR conditions)}

The physical conditions of regime 2 are already characteristic of the PCTR but, as in the prominence regime and because of the positive value of parameter $\alpha$, no thermal instability appears and the heating mechanisms do not produce any important difference in the period, damping time and damping per period for slow and fast waves. For this reason, in Fig. 5 we have only considered a constant heating per unit volume, and the overall behaviour of the three parameters is very similar to that of the prominence regime but with the slow wave having a maximum value of the damping per period slightly larger than in the
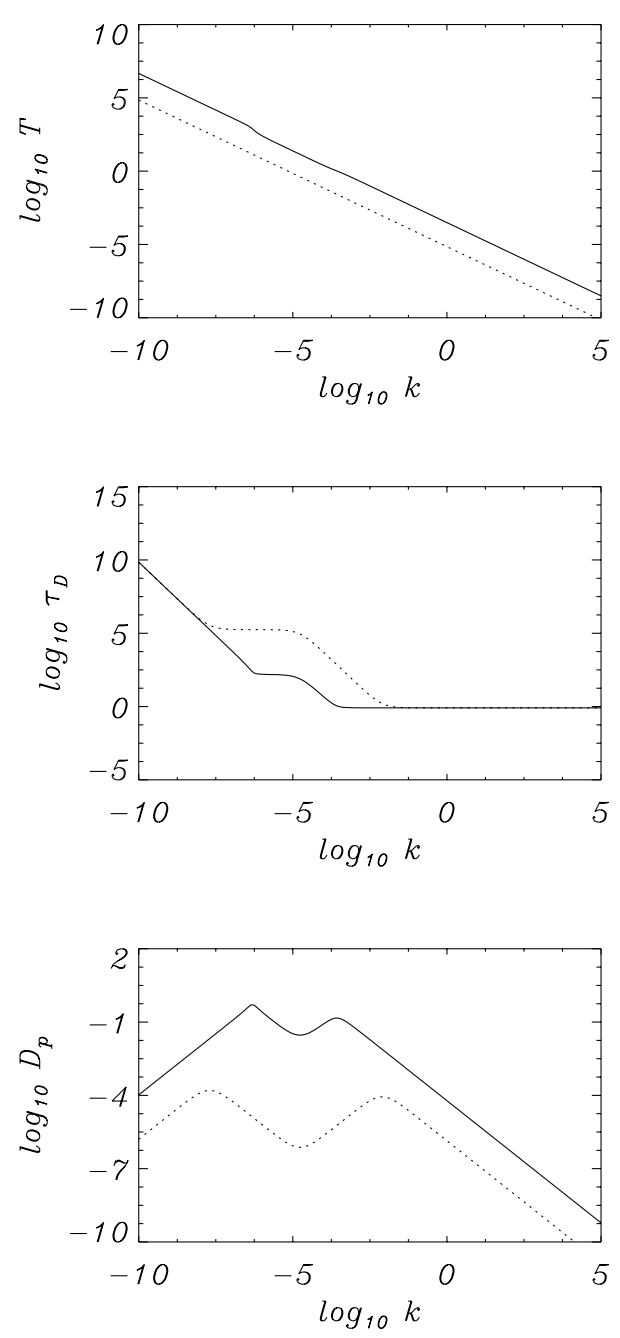

Fig. 5. Period $(T)$, damping time $\left(\tau_{\mathrm{D}}\right)$ and damping per period $\left(D_{\mathrm{p}}\right)$ for the fast (dotted) and slow (continuous) waves in the PCTR regime 2, for the heating mechanism characterised by $a=b=0$.

case of the prominence regime, which means that for a certain interval of wavenumbers slow waves are heavily damped.

\subsection{Regimes 3 and 4 (PCTR conditions)}

The physical conditions of regimes 3 and 4 also belong to the PCTR, with regime 3 a border case between regimes 1 and 2, on one hand, and regimes 4 and 5 on the other hand. The reason is that thermal instability starts to appear in this regime. In the case of constant heating per unit mass and heating by Alfvén mode/mode conversion, no instabilities appear, while in the case of heating by coronal current and heating by Alfvén mode/anomalous conduction damping, thermal instability appears as well as unstable wave modes; finally, in the case of constant heating per unit volume, thermal instability appears while wave modes are stable.

The apparition of these instabilities can be understood following the instability criteria, for wave and thermal condensation modes, pointed out by Field (1965). These criteria are: the isobaric criterion

$L_{T}-\frac{\rho_{0}}{T_{0}} L_{\rho}<0$ 

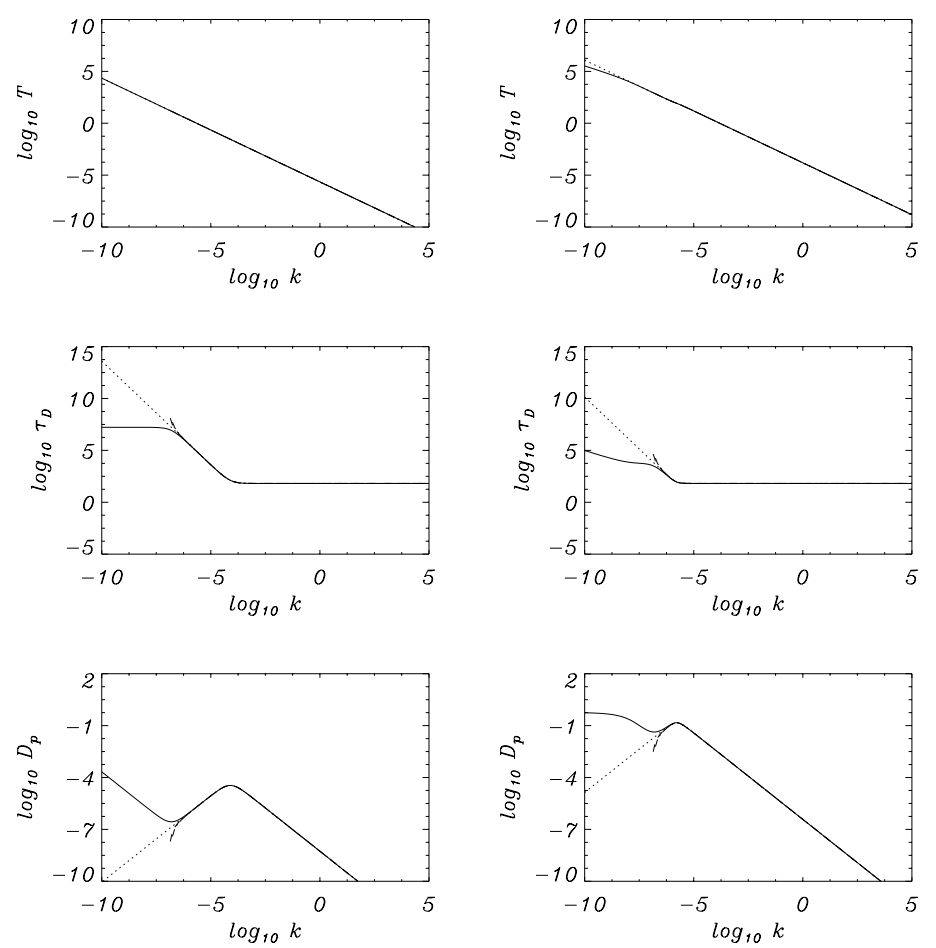

Fig. 6. Period $(T)$, damping time $\left(\tau_{\mathrm{D}}\right)$ and damping per period $\left(D_{\mathrm{p}}\right)$ for the fast (left) and slow (right) waves in the PCTR regime 3. Continuous, dotted, dashed and dash-dotted lines correspond to the heating mechanisms characterised by: $a=b=0 ; a=1, b=0 ; a=b=1 ; a=b=7 / 6$, respectively.

which, when satisfied, indicates the instability of the thermal or condensation mode, and the isentropic criterion

$L_{T}-\frac{\rho_{0}}{(\gamma-1) T_{0}} L_{\rho}<0$

which, when satisfied, indicates instability of the wave modes. For instance, in regime 3 and in the case of constant heating per unit mass $(a=1, b=0)$ :

$L(\rho, T)=\chi^{*} \rho-h \rho$,

so that

$L_{\rho}=\chi^{*}-h=0$

and

$L_{T}=0$.

Then, appliying the above criteria no instability appears either for the thermal or wave modes. When we consider constant heating per unit volume $(a=b=0)$, then

$L_{T}=0, L_{\rho}=\chi^{*}>0$.

Now, applying the above criteria we can see that the isobaric criterion is satisfied, therefore thermal instability appears, while the isentropic criterion is not satisfied and the wave modes are stable. Finally, in the case of coronal current dissipation $(a=b=1)$

$L_{\rho}=\chi^{*}-h T=0$

and

$L_{T}=-h \rho<0$.
Then, from the above criteria, we have thermal as well as wave instabilities.

The above results point out that, in regime 3 , the heating mechanisms are important in order to determine when thermal or wave instabilities appear. On the other hand, for a certain wavenumber the instabilities disappear due to the stabilising effect of thermal conduction which, of course, depends on the angle of propagation $\theta$. Using the approximation considered by Field (1965), a critical wavenumber can be computed (Eqs. (60) and (26) of Field's paper) which, in many cases, constitutes a good approximation to the actual wavenumber at which stabilisation is attained. For instance, in regime 3 and in the case of heating by Alfvén mode/mode conversion the stabilisation of the wave modes is obtained at $\log k=-6.95$ while Field's critical wavenumber gives a value of $\log k=-6.82$. Finally, when thermal as well as wave mode instabilities are present, the growth time for wave modes is much greater than the growth time of the thermal instability.

In Fig. 6, we can see that while the period of fast waves is not affected, the behaviour of the period of slow waves for wavenumbers between $10^{-15}$ and $10^{-10} \mathrm{~m}^{-1}$ is different for the constant heating per unit mass mechanism. Also, in the case of the damping time and damping per period, the influence of the heating mechanisms is clearly seen. Because of the presence of wave instabilities, for two of the considered heating mechanisms the damping time and the damping per period have not been plotted for the whole range of wavenumbers considered. Furthermore, the global behaviour of the damping time and damping per period is very different from that of regimes 1 and 2, although, in general, the damping time is smaller for 

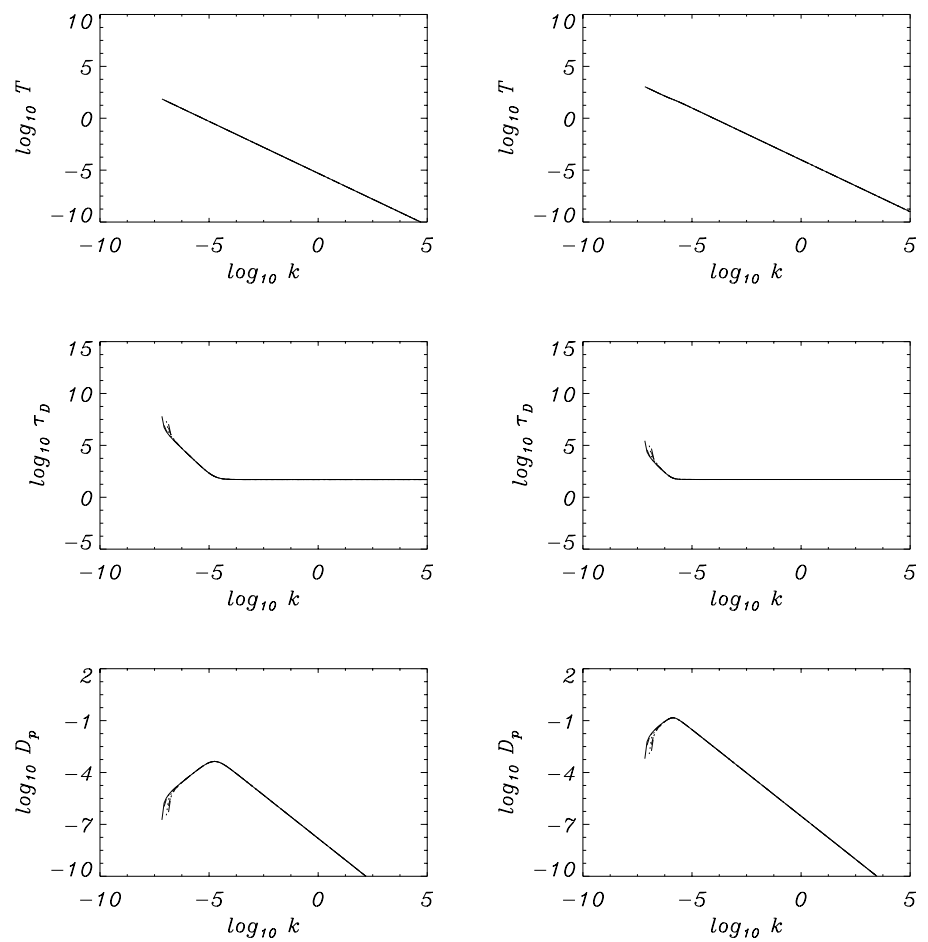

Fig. 7. Period $(T)$, damping time $\left(\tau_{\mathrm{D}}\right)$ and damping per period $\left(D_{\mathrm{p}}\right)$ for the fast $($ left $)$ and slow (right) waves in the PCTR regime 4 . The continuous line corresponds to the heating mechanism characterised by $a=b=0$. The plots corresponding to the five mechanisms overlap except close to $k=10^{-7} \mathrm{~m}^{-1}$.

slow than for fast waves while the damping per period is always greater for slow waves. Finally, in the region from small to large wavenumbers, the damping time is almost constant and independent of the considered heating mechanism.

In the case of regime 4 , for all the different considered heating mechanisms thermal as well as wave modes instabilities appear. In Fig. 7, the region of wavenumbers between $10^{-10}$ and $10^{-7} \mathrm{~m}^{-1}$, where no curves are shown, is where thermal and wave instabilities appear. Outside from the instability region the damping time, for whatever heating mechanism considered, rapidly becomes almost independent of the wavenumber.

\section{Regime 5 (coronal conditions)}

In regime 5, corresponding to coronal conditions, instabilities appear again. In the case of constant heating per unit mass, coronal current dissipation heating and heating by Alfvén mode/mode conversion, thermal as well as wave mode instabilities appear, while for the rest of heating mechanisms, only the thermal instability are present. Then, again, heating mechanisms play a key role for the presence or not of wave modes instabilities. In Fig. 8 we have plotted the period, damping time and damping per period for the different heating mechanisms. An interesting feature is that for those heating mechanisms in which no instabilities appear, the general shape of the damping time curve is similar to that of regimes 1 and 2, with two plateaus, but in this case the second one corresponds to a greater damping time than in those regimes. Furthermore, looking to the plots corresponding to regimes 3 and 4 , a similar behaviour of the damping time versus the wavenumber can be suspected because the final part of the curve is similar in all these regimes.

Finally, and as a summary of the behaviours found in our analysis, Fig. 9 shows the period, damping time and damping per period for the five regimes when a constant heating per unit mass mechanism is taken into account. From the inspection of the figure, we can conclude that the behaviours, in particular, of the damping time and the damping per period change significantly when going from very small to large wavenumbers. For small and large wavenumbers, the damping time in regimes 3, 4 and 5 becomes almost constant and the same happens, for large wavenumbers, for regimes 1 and 2. Also, we can see in this figure that in the region of large wavenumbers the damping time, for a fixed wavenumber, increases from prominence to coronal regime.

\section{Conclusions}

In this paper, our main aim has been to analyze the time damping of linear magnetoacoustic waves by the joint effect of optically thin radiation, thermal conduction and a variety of heating terms. To this end, we have solved numerically the full dispersion relation for linear non-adiabatic MHD waves in an unbounded magnetised plasma and have studied the behaviour of the period, damping time and damping per period of the magnetoacoustic waves in media having physical properties akin to those of solar prominences, PCTR and solar corona.

The main conclusions which can be extracted from our study are:

1.- The consideration of different prominence regimes, distinguished by the values of parameters $\alpha$ and $\chi^{*}$, does not 

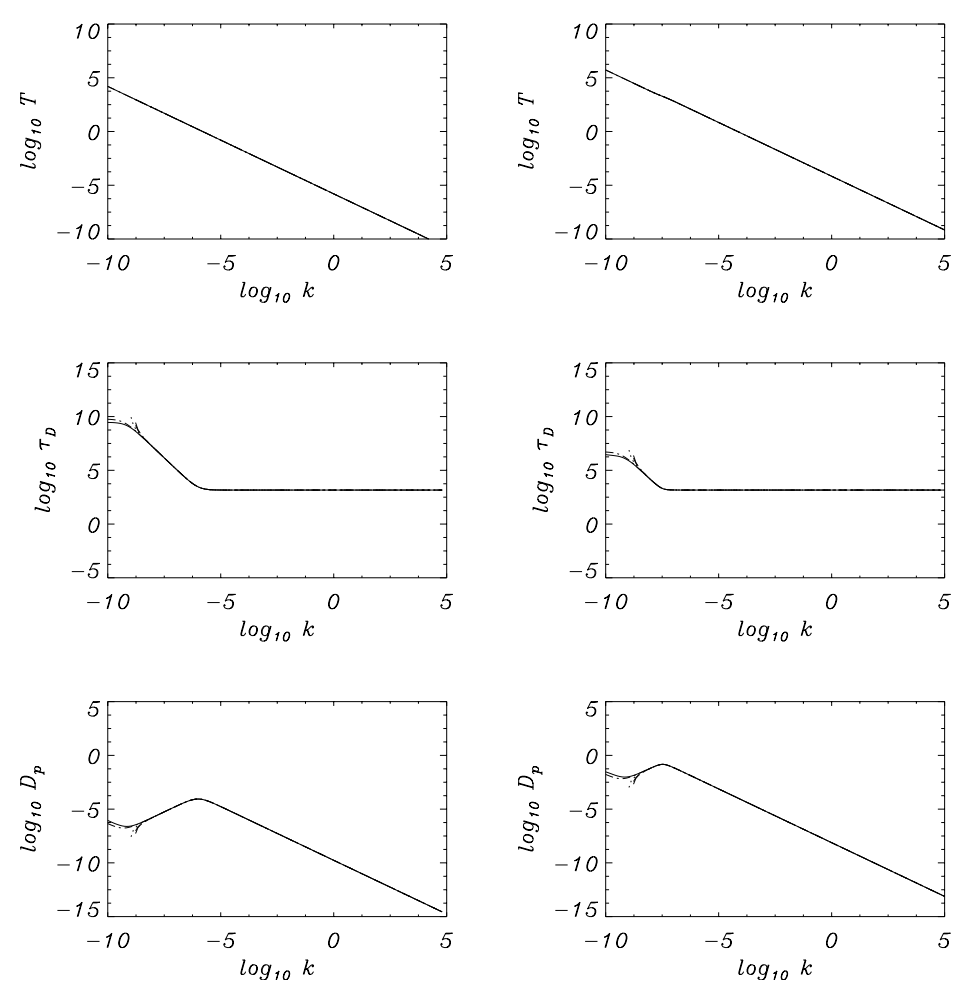

Fig. 8. Period $(T)$, damping time $\left(\tau_{\mathrm{D}}\right)$ and damping per period $\left(D_{\mathrm{p}}\right)$ for the fast (left) and slow (right) waves in regime 5 (coronal regime). Continuous, dotted, dashed, dash-dotted and dash-three dotted lines correspond to the heating mechanisms characterised by: $a=b=0 ; a=1$, $b=0 ; a=b=1 ; a=0.5, b=-0.5, a=b=7 / 6$, respectively.

produce important differences in the behaviour of the period of the waves, while the damping time and the damping per period are modified. In particular, it can be seen that the values of the the parameters $\alpha$ and $\chi^{*}$, which try to represent the case of an optically thick prominence, produce a substantial decrease in the damping time of fast and slow waves in the region of wavenumbers between $10^{-10}$ and $10^{-7} \mathrm{~m}^{-1}$, while the opposite happens in the region of wavenumbers between $10^{-7}$ and $10^{-3} \mathrm{~m}^{-1}$.

2.- In the different prominence regimes considered, as well as in regime 2 (PCTR regime), the different heating mechanisms do not produce any noticeable effect on the quantities under study.

3.- In regimes 1 and 2 we find very large damping times for very small wavenumbers, almost constant damping time for intermediate wavenumbers, followed by a decrease and, again, almost constant damping times for large wavenumbers. This pattern describes the overall behaviour in these regimes for all the heating mechanisms.

4.- When regime 3 is considered, thermal as well as wave instabilities start to appear. This regime can be considered a pathological one since the value of the parameter $\alpha$ is zero, separating regimes with $\alpha$ positive from those with $\alpha$ negative, in which thermal instabilities are expected. Furthermore, in this regime the importance of the heating mechanism considered in the apparition of wave instabilities becomes evident. This effect can be easily understood following the instability criteria introduced by Field (1965). On the other hand, the behaviour of the damping time and damping per period is different from regimes 1 and 2, and starting from a wavenumber around $10^{-5} \mathrm{~m}^{-1}$, the damping time becomes almost wavenumber independent for all the considered heating mechanisms.

5.- In regime 4, another PCTR situation, thermal as well as wave instabilities appear for all the heating mechanisms. In this case the key role is played by the parameter $\alpha$, whose value is -2.5 , and, then, for very small wavenumbers we have instabilities which disappear once a certain wavenumber is attained. Again, such as happens with regime 3, the time damping becomes almost constant for small and large wavenumbers.

6.- In regime 5 , i.e. coronal conditions, the value of the parameter $\alpha$ is -1 and again for some heating mechanisms we have wave and thermal instabilities, while for the rest we only have thermal instabilities. Then, the heating mechanisms are again determinant for the presence or not of wave instabilities.

7.- In general, the damping per period is very small for very small and large wavenumbers, which means that many periods are needed to damp the waves. However, there is a range of intermediate wavenumbers for which the damping per period, for slow waves, varies between 1 and $10^{-2}$, say, so 1-100 periods are needed to damp the wave. In particular, the maximum damping per period for slow waves is always two orders of magnitude larger than that of fast waves.

8.- A general belief is that the thermally induced damping time is always greater for fast waves than for slow waves. From our study, we can conclude that the wavenumber plays a key role and that for very small and very large wavenumbers the damping times are equal for both waves, while in an intermediate region they are different, with the fast wave damping time greater than the slow wave one (see Figs. 2 and 8). 

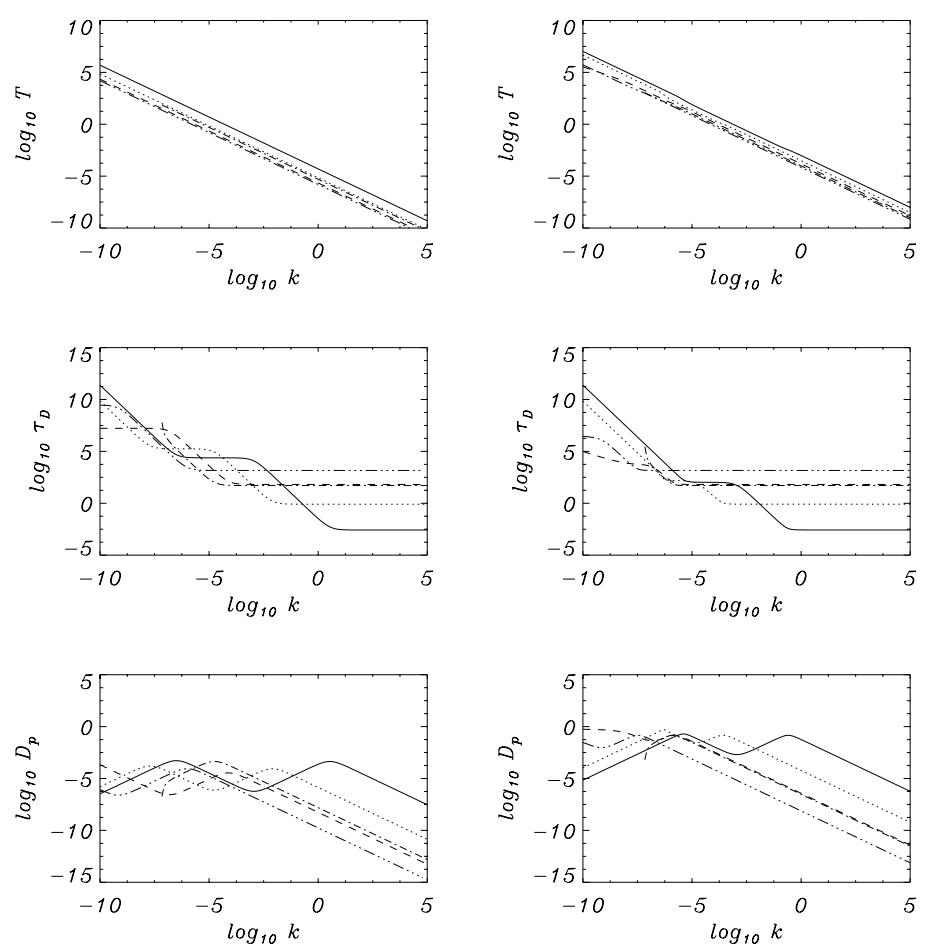

Fig. 9. Period $(T)$, damping time $\left(\tau_{\mathrm{D}}\right)$ and damping per period $\left(D_{\mathrm{p}}\right)$ for the fast (left) and slow (right) waves for the five regimes considered and a constant heating per unit mass $(a=b=0)$. Continuous, dotted, dashed, dash-dotted and dash-three dotted lines correspond to regime 1, regime 2 , regime 3 , regime 4 and regime 5 , respectively.

9.- In all the considered cases, and as a general rule, small wave periods, for large wavenumbers, and large periods, for very small wavenumbers, are found for both the fast and slow waves.

10.- An observational fact is that wavelengths of perturbations detected in prominences and in the surrounding solar corona can vary from a few hundreds of $\mathrm{km}$ (or even less) to tens of thousands of $\mathrm{km}$, i.e. $k \approx 10^{-5}-10^{-10} \mathrm{~m}^{-1}$. Then, Fig. 9 summarizes, for the different regimes considered and a particular heating mechanism, the variety of behaviours which appear within the above quoted wavenumber interval. It can be seen that, except for the period, the magnitudes of the damping time and damping per period vary by a substantial amount within such wavenumber interval.

On the other hand, although it could be tempting to apply these results to coronal seismology, it would be meaningless since no particular structure has been taken into account. This application will be the subject of forthcoming papers in which we will consider particular coronal structures such as bounded magnetic slabs representing the prominence-corona medium, or cylindrical flux tubes, with cool and hot parts, representing the fibril structure of prominences. Also, many effects which could be of interest for coronal seismology have not been considered here. For instance, non-linear effects which have been taken into account in the study of the dissipation of slow MHD waves in coronal plumes (Ofman et al. 1999) or in the study of magnetoacoustic waves in optically thin quasientropic plasmas waves (Nakariakov et al. 2000b); or the effect of the 2D and 3D structure, which is relevant when particular coronal structures are taken into account (such as the 2D numerical simulations of magnetoacoustic waves in polar plumes by Ofman et al. 2000b); or kinetics effects in waves associated to the solar wind or in coronal holes. The main reason for not taking into account such effects in our study is that we are mostly interested in the understanding of small amplitude oscillations in coronal structures, such as prominences, and in ascertaining the joint effect of optically thin radiation, thermal conduction and different heating terms in the damping time of those oscillations.

Acknowledgements. The authors acknowledge the financial support received from MCyT under grant AYA2003-00123.

\section{Appendix A}

In what follows, overline means complex conjugate.

Theorem. Given $p(z)=a_{0}+a_{1} z+\cdots+a_{n} z^{n}$, and $b_{j} \in R$, $j=0, \ldots, n$.

(a) If $a_{2 k+1}=b_{2 k+1}, a_{2 k}=\mathrm{i} b_{2 k}$, then $p(-\bar{z})=-\overline{p(z)}$.

(b) If $a_{2 k+1}=\mathrm{i} b_{2 k+1}, a_{2 k}=b_{2 k}$, then $p(-\bar{z})=\overline{p(z)}$.

Proof. (a) If we write $p(z)=\sum_{j=0}^{n} a_{j} z^{j}=\sum a_{2 k} z^{2 k}+$ $\sum a_{2 k+1} z^{2 k+1}$, then using elementary properties of complex analysis we have:

$$
\begin{aligned}
p(-\bar{z}) & =\sum a_{2 k}(-\bar{z})^{2 k}+\sum a_{2 k+1}(-\bar{z})^{2 k+1} \\
& =\sum a_{2 k} \bar{z}^{2 k}-\sum a_{2 k+1} \bar{z}^{2 k+1} \\
& =\sum \mathrm{i} b_{2 k} \bar{z}^{2 k}-\sum b_{2 k+1} \bar{z}^{2 k+1} \\
& =\sum \mathrm{i} b_{2 k} \overline{z^{2 k}}-\sum b_{2 k+1} \overline{z^{2 k+1}}
\end{aligned}
$$


Since $b_{j} \in \boldsymbol{R}$, then $\bar{b}_{j}=b_{j}$, and from above:

$$
\begin{aligned}
p(-\bar{z}) & =\sum \overline{-\mathrm{i} b_{2 k}} \overline{z^{2 k}}-\sum \overline{b_{2 k+1}} \overline{z^{2 k+1}} \\
& =-\sum \overline{\mathrm{i} b_{2 k} z^{2 k}}-\sum \overline{b_{2 k+1} z^{2 k+1}} \\
& =-\left(\overline{\sum \mathrm{i} b_{2 k} z^{2 k}+\sum b_{2 k+1} z^{2 k+1}}\right) \\
& =-\left(\overline{\sum a_{2 k} z^{2 k}+\sum a_{2 k+1} z^{2 k+1}}\right)=-\overline{p(z)} .
\end{aligned}
$$

(b) Similarly,

$$
\begin{aligned}
& p(-\bar{z})=\sum a_{2 k}(-\bar{z})^{2 k}+\sum a_{2 k+1}(-\bar{z})^{2 k+1} \\
& =\sum a_{2 k} \bar{z}^{2 k}-\sum a_{2 k+1} \bar{z}^{2 k+1} \\
& =\sum b_{2 k} \overline{z^{2 k}}-\sum \mathrm{i} b_{2 k+1} \overline{z^{2 k+1}} \\
& =\sum \overline{b_{2 k}} \overline{z^{2 k}}-\sum \overline{-\mathrm{i} b_{2 k+1}} \overline{z^{2 k+1}} \\
& =\sum \overline{b_{2 k} z^{2 k}}-\sum \overline{-i b_{2 k+1} z^{2 k+1}} \\
& =\sum \overline{b_{2 k} z^{2 k}}+\sum \overline{\mathrm{i} b_{2 k+1} z^{2 k+1}} \\
& =\overline{\sum b_{2 k} z^{2 k}+\sum \mathrm{i} b_{2 k+1} z^{2 k+1}} \\
& =\overline{\sum a_{2 k} z^{2 k}+\sum a_{2 k+1} z^{2 k+1}}=\overline{p(z)} \text {. }
\end{aligned}
$$

Corollary. Given $p(z)$ a polynomial which verifies the above conditions $(a)$ or $(b)$.

(a) If $z=\omega$ is a root of the polynomial, then $z=-\bar{\omega}$ is also $a$ root.

(b) When the polynomial is of odd order, it always has a purely imaginary root.

Proof. (a) We know that $p(\omega)=0$ and using the above theorem we have

$$
p(-\bar{\omega})=\left\{\begin{array}{l}
-\overline{p(\omega)}=-\overline{0}=0 \text { or } \\
p(\omega)=\overline{0}=0
\end{array}\right.
$$

(b) Since $p(z)$ has $n$ roots and $n$ is odd, then from (a) one of the roots must verify $\omega=-\bar{\omega}$, so $\omega+\bar{\omega}=0$. Hence, $2 \operatorname{Re} \omega=0$ which implies that $\operatorname{Re} \omega=0$.

\section{References}

Aschwanden, M., Fletcher, L., Schrijver, C. J., \& Alexander, D. 1999, ApJ, 520, 880
Banerjee, D., Hasan, S. S., \& Christensen-Dalsgaard, J. 1997, Sol. Phys., 172, 52

Banerjee, D., O’Shea, E., \& Doyle, J. G. 2000, Sol. Phys., 196, 63

Banerjee, D., O'Shea, E., Doyle, J. G., \& Goosssens, M. 2001, A\&A, 380, L39

Bünte, M., \& Bogdan, T. 1994, A\&A, 283, 642

Dahlburg, R. B., \& Mariska, J. T. 1988, Sol. Phys., 117, 51

DeForest, C. E., \& Gurman, J. 1998, ApJ, 501, L217

De Moortel, I., Ireland, J., \& Walsh, R. W. 2000, A\&A, 355, L23

Engvold, O. 2001, in INTAS Workshop on MHD Waves in Astrophysical Plasmas, ed. J. L. Ballester, \& B. Roberts, Universitat de les Illes Balears, Spain

Field, G. B. 1965, ApJ, 142, 531

Hildner, E. 1974, Sol. Phys., 35, 123

Ibañez, M. H., \& Escalona, O. B. 1993, ApJ, 415, 335

Kliem, B., Dammasch, I. E., Curdt, W., \& Wilhelm, K. 2002, ApJ, 568, L61

Mihalas, D., \& Mihalas, B. W. 1984, in Foundations of Radiation Hydrodynamics (New York: Oxford University Press)

Milne, A. M., Priest, E. R., \& Roberts, B. 1979, ApJ, 232, 304

Molowny-Horas, R., Wiehr, E., Balthasar, H., Oliver, R., \& Ballester, J. L. 1999, JOSO Annual Report 1998, Astronomical Institute Tatranska Lomnica, 126

Nakariakov, V. M., Ofman, L., De Luca, E., Roberts, B., \& Davila, J. M. 1999, Science, 285, 862

Nakariakov, V. M., Ofman, L., \& Arber, T. D. 2000a, A\&A, 353, 741

Nakariakov, V. M., Mendoza-Briceño, C. A., \& Ibañez, S. M. H. 2000b, ApJ, 528, 767

Ofman, L., \& Wang, T. 2002, ApJ, 580, L85

Ofman, L., Romoli, M., Poletto, G., Noci, G., \& Kohl, J. L. 1997, ApJ, 491, L111

Ofman, L., Nakariakov, V. M., \& DeForest, C. E. 1999, ApJ, 514, 441

Ofman, L., Romoli, M., Poletto, G., Noci, G., \& Kohl, J. L. 2000a, ApJ, 529, 592

Ofman, L., Nakariakov, V. M., \& Sehgal, N. 2000b, ApJ, 533, 1071

Porter, L. J., Klimchuk, J. A., \& Sturrock, P. A. 1994a, ApJ, 435, 482

Porter, L. J., Klimchuk, J. A., \& Sturrock, P. A. 1994b, ApJ, 435, 502

Robbrecht, E., Verwichte, E., Berghmans, D., et al. 2001, A\&A, 370, 591

Rosner, R., Tucker, W. H., \& Vaiana, G. S. 1978, ApJ, 220, 643

Terradas, J., Oliver, R., \& Ballester, J. L. 2001, A\&A, 378, 635

Terradas, J., Molowny-Horas, R., Wierh, E., et al. 2002, A\&A, 393, 637

Wang, T., Solanki, S. K., Curdt, W., Innes, D., \& Dammasch, I. E. 2002, ApJ, 574, L101

Webb, A. R., \& Roberts, B. 1980, Sol. Phys., 68, 71 